\title{
Performance evaluation on GNSS, wheel speed sensor, yaw rate sensor, and gravity sensor integrated positioning algorithm for automotive navigation system
}

\author{
Joong-hee $\operatorname{Han}^{1,{ }^{*}}$ and Chi-ho Park ${ }^{1}$ \\ ${ }^{1}$ Intelligent Device and Systems Research Group, DGIST, Republic of Korea
}

\begin{abstract}
The Global Navigation Satellite System (GNSS) positioning technique is widely used for the automotive navigation system since it can provide the stable and accurate position and velocity in the most road environments at an affordable price. However, the performance of GNSS positioning technique is degraded in certain areas, where GNSS signals are blocked by buildings and tunnel. To overcome this problem, the GNSS positioning technique should be integrated with dead reckoning (DR) sensors such as accelerometer, gyroscope, and odometer. Recently, the most passenger cars are equipped with the Advanced Driver Assistance System (ADAS) based on numerous sensors to improve safety and convenience in driving. Among sensors for the ADAS, vehicle dynamic sensors such as wheel speed sensor (WSS), yaw rate sensor (YRS), gravity sensor (GS) can be used for the DR algorithm since those sensors measure vehicle's motions. Therefore, this paper evaluates the vehicle positioning algorithm that integrate the GNSS with a threedimensional dead reckoning based on WSS, YRS, and GS. The vehicle positioning algorithm is implemented through the extended Kalman filter of a loosely-coupled mode. Performance was evaluated through tests carried out in real driving trajectory including various GNSS signals reception environments. It is found that the proposed algorithm can be an alternative solution to compensate the limitation of the GNSS positioning technique, without the use of a low-cost inertial measurement unit.
\end{abstract}

\section{Introduction}

The importance of accuracy, integrity, and continuity in a positioning technique has increasingly been emphasized in land vehicle application such as automotive navigation, advanced driver assistance systems (ADAS), intelligent transportation system (ITS) [1-2]. Nowadays, global navigation satellite system (GNSS) positioning technique is widely used for these applications since it can provide the stable and accurate position and velocity in the most road environments at an affordable price. However, the GNSS positioning technique may produce abnormal position errors mainly cause by multipath and non-line-of-sight (NLOS) signal interferences in urban canyons. Therefore, the standalone GNSS positioning technique cannot guarantee an accurate and continuous positioning in all vehicle driving environment [3]. To overcome this problem, the GNSS positioning technique should be integrated with additional sensors such as inertial measurement unit (IMU) and odometer.

Recently, to improve safety and convenience in driving, most passenger car have been equipped with various advanced driver assistance system (ADAS) applications such as anti-lock braking system (ABS), electronic stability control (ESC), and traction control system(TCS) [4]. ADAS uses data which are continuously acquired by numerous on-board vehicle sensors such as wheel speed sensor (WSS), yaw rate sensor (YRS), gravity sensor (GS), steering angle sensor (SAS). These sensors measure and provide vehicle's motions. Therefore, on-board vehicle sensors can be applied to develop for the car positioning technique. The development of car positioning technique can be used by on-board vehicle sensors following two main strategies. First, on-board vehicle sensors are applied to compensate for the INS error. Most of the previous researches are the integration of WSS with GNSS/INS to prevent INS error degradation during GNSS outages [5-7] Reference [8] developed five types of sensors integration algorithms for precise positioning, namely GPS/INS/WSS, GPS/INS/GS/YRS, GPS/INS/SAS, GPS/INS/WSS/SAS, and GPS/INS/WSS/GL/YRS/SAS. Second, on-board vehicle sensors are applied for dead reckoning (DR) to overcome the limitation of GNSS technique. Most of the related researches developed integration of GNSS with the deadreckoning on-board vehicle sensors in two ways, GNSS/WSS as well as GNSS/WSS/YRS [9-11]. Also, GNSS/on-board vehicle sensors integration based on vehicle model was studied [12-13].

This paper proposes and evaluates an EKF (Extended Kalman Filter)-based GNSS/on-board sensors integrated vehicle positioning algorithm to overcome the limitation of GNSS based technique. This combines data from a

* Corresponding author: jhhan@dgist.ac.kr 
GNSS receiver with data coming from six on-board vehicle sensors: two WSS, a YRS, a lateral GS, and a longitudinal GS. A description of the proposed algorithm is presented in Section 2. A performance evaluation of the proposed algorithm is provided in Section 3. The conclusion is given in Section 4.

\section{GNSS/on-board vehicle sensors integration algorithm}

Figure 1 is a block diagram of the GNSS/on-board vehicle sensors integration algorithm. The GNSS/on-board sensors integrated vehicle positioning algorithm is implemented loosely coupled integration through EKF. Three dimensional DR based on on-board vehicle sensors calculates the positions, the velocities in the navigation frame, and attitudes at an interval of $0.02 \mathrm{~s}$. A three dimensional DR based on on-board vehicle sensors uses data measuring from two rear WSS, a YRS, a lateral GS, and a longitudinal GS. The procedure for this DR is as follows. To define the velocity in the body frame using the vehicles speed derived from two rear WSS we applied two no holonomic constraints on the lateral and vertical directions. In additional, the center of body frame is set to the center of rear wheel axle. The velocities in the body frame are defined as follows:

$$
\boldsymbol{v}^{\boldsymbol{b}}=\left[\begin{array}{l}
v_{x} \\
v_{y} \\
v_{z}
\end{array}\right]=\left[\begin{array}{c}
\left(v_{r L}+v_{r R}\right) / 2 \\
0 \\
0
\end{array}\right]
$$

where $v_{x}, v_{y}$, and $v_{z}$ are velocities in the body frame, $v_{r L}$ and $v_{r R}$ are the rear left and rear right WSS measurements, respectively.

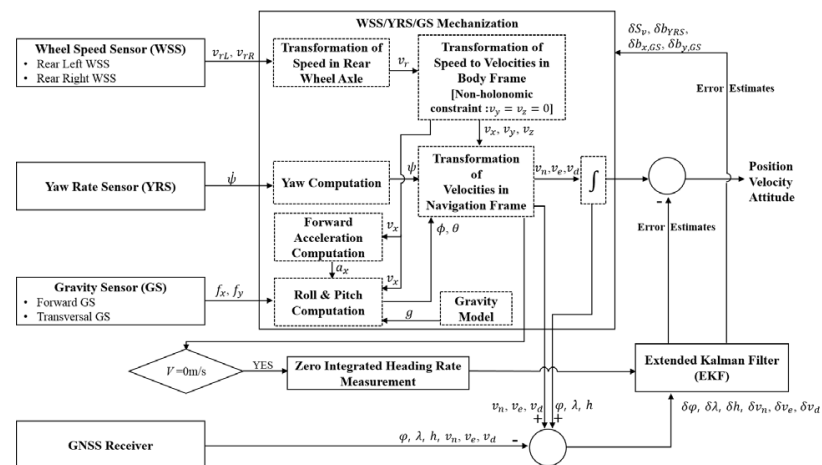

Fig. 1. Block diagram of the GNSS/on-board vehicle sensors integration algorithm.

When the forward vehicle acceleration is derived from WSS measurement, the pitch angle $(\theta)$ can be computed as shown in Equation (2) using the longitudinal GS and Earth's gravity model.

$$
\theta=\sin ^{-1}\left(\frac{f_{x}-a_{x}}{g}\right)
$$

where $f_{x}$ is the longitudinal GS measurement, $a_{x}$ is the WSS derived foward vehicle acceleration, $g$ is the gravity at the present location.
The roll angle $(\phi)$ can be calculated as shown in Equation (3) using the lateral GS, YRS, Earth's gravity model and the value for forward velocity.

$$
\phi=\sin ^{-1}\left(\frac{f_{y}-v_{x} \dot{\psi}}{g \cos \theta}\right)
$$

where $f_{y}$ is the lateral GS measurement, $v_{x}$ is the foward velocity, $\dot{\psi}$ is the YRS measurement, and $g$ is the gravity at the present location.

The yaw angle $(\Psi)$ can be calculated by numerical integration using the YRS, as expressed in

$$
\psi(\mathrm{t})=\psi(\mathrm{t}-1)+\dot{\psi} \Delta \mathrm{t}
$$

where $\psi(\mathrm{t})$ and $\psi(\mathrm{t}-1)$ are the yaw angle at time $\mathrm{t}$ and $\mathrm{t}-1$, respectively, $\dot{\psi}$ is the YRS measurement, and $\Delta \mathrm{t}$ is the samling time interval.

The velocities in the navigation frame can be transformed by using

$$
\boldsymbol{v}^{\boldsymbol{n}}=\left[\begin{array}{l}
v_{n} \\
v_{e} \\
v_{d}
\end{array}\right]=\boldsymbol{C}_{\boldsymbol{b}}^{\boldsymbol{n}} \boldsymbol{v}^{\boldsymbol{b}}
$$

where $v_{n}, v_{e}$, and $v_{d}$ are velocities in the navigation frame, and $\boldsymbol{C}_{\boldsymbol{b}}^{\boldsymbol{n}}$ is the direction cosine matrix from the body frame to the navigation frame.

The velocities in the navigation frame are transformed into the rate of change of the positions in the geodetic coordinate system by using Equation (6), and then integrated over the sample interval to obtain positions in the geodetic coordinates system.

$$
\dot{\boldsymbol{r}}^{n}=\left[\begin{array}{c}
\dot{\varphi} \\
\dot{\lambda} \\
\dot{h}
\end{array}\right]=\left[\begin{array}{ccc}
\frac{1}{M+h} & 0 & 0 \\
0 & \frac{1}{(N+h) \cos \varphi} & 0 \\
0 & 0 & -1
\end{array}\right]\left[\begin{array}{l}
v_{n} \\
v_{e} \\
v_{d}
\end{array}\right]
$$

where $\dot{\varphi}, \dot{\lambda}$, and $\dot{h}$ are the rate of change of the latitude, longitude, and ellipsoidal height, $M$ is the radius of curvature of the meridian, $N$ is the radius curvature of the prime vertical, $h$ is the ellipsoidal height, $\varphi$ is the geodetic latitude.

In EKF-based GNSS/on-board sensors integrated vehicle positioning algorithm, the state vector of the navigation error is composed of latitude error $(\delta \varphi)$, longitude error $(\delta \lambda)$, ellipsoidal height error $(\delta h)$, north velocity error $\left(\delta v_{n}\right)$, east velocity error $\left(\delta v_{e}\right)$, down velocity error $\left(\delta v_{d}\right)$, roll error $(\delta \phi)$, pitch error $(\delta \theta)$, and yaw error $(\delta \psi)$. The state vector of the sensor error is composed of the average of left and right rear WSS scale factor $\left(\delta s_{v}\right)$, the YRS bias $\left(\delta b_{Y R S}\right)$, the longitudinal GS bias $\left(\delta b_{x, G S}\right)$, and lateral GS bias $\left(\delta b_{y, G S}\right)$, defined random constants. The dynamic model of GNSS/on-board sensors integrated vehicle positioning algorithm is shown in the following Equation (7):

$$
\boldsymbol{\delta} \dot{\boldsymbol{x}}=\boldsymbol{F} \boldsymbol{\delta} \boldsymbol{x}+\boldsymbol{G u}
$$

where $\boldsymbol{F}$ is the dynamic matrix, $\boldsymbol{\delta} \boldsymbol{x}$ is the error state vector, $\boldsymbol{G}$ is the shaping matrix, $\boldsymbol{u}$ is the white noise vector.

The error state vector $(\boldsymbol{\delta} \boldsymbol{x})$ is written as follows : 
$\boldsymbol{\delta} \boldsymbol{x}=\left[\delta \varphi \delta \lambda \delta h \delta v_{n} \delta v_{e} \delta v_{d} \delta \phi \delta \theta \delta \psi \delta s_{v} \delta b_{Y R S} \delta b_{x, G S} \delta b_{y, G S}\right]^{T}(8)$

The dymamic matrix $(\boldsymbol{F})$ is written as follows :

$$
\boldsymbol{F}=\left[\begin{array}{cccc}
\boldsymbol{F}_{11} & \boldsymbol{F}_{12} & \boldsymbol{0}_{3 \times 3} & \boldsymbol{0}_{3 \times 4} \\
\mathbf{0}_{3 \times 3} & \mathbf{0}_{3 \times 3} & \boldsymbol{F}_{23} & \boldsymbol{F}_{24} \\
\boldsymbol{0}_{3 \times 3} & \mathbf{0}_{3 \times 3} & \mathbf{0}_{3 \times 3} & \boldsymbol{F}_{34} \\
\mathbf{0}_{4 \times 3} & \mathbf{0}_{4 \times 3} & \mathbf{0}_{4 \times 3} & \mathbf{0}_{4 \times 4}
\end{array}\right]
$$

where $\mathbf{0}_{\boldsymbol{n} \times \boldsymbol{m}}$ is $\mathrm{n} \times \mathrm{m}$ zero matrix and $\boldsymbol{F}_{\mathbf{1 1}}, \boldsymbol{F}_{\mathbf{1 2}}, \boldsymbol{F}_{\mathbf{2 3}}, \boldsymbol{F}_{\mathbf{2 4}}$, and $\boldsymbol{F}_{\mathbf{3 4}}$ are written as follows:

$$
\begin{gathered}
\boldsymbol{F}_{\mathbf{1 1}}=\left[\begin{array}{ccc}
0 & 0 & \frac{-v_{n}}{(M+h)^{2}} \\
\frac{v_{e} \tan \varphi}{(N+h) \cos \varphi} & 0 & \frac{-v_{e}}{(N+h)^{2} \cos \varphi} \\
0 & 0 & 0
\end{array}\right] \\
\boldsymbol{F}_{\mathbf{1 2}}=\left[\begin{array}{ccc}
\frac{1}{M+h} & 0 & 0 \\
0 & \frac{1}{(N+h) \cos \varphi} & 0 \\
0 & 0 & -1
\end{array}\right] \\
\boldsymbol{F}_{\mathbf{2 3}}=\left[\begin{array}{llll}
0 & -v_{x} \sin \theta \cos \psi & -v_{x} \cos \theta \sin \psi \\
0- & v_{x} \sin \theta \sin \psi & v_{x} \cos \theta \cos \psi \\
0-v_{x} \cos \theta & & 0
\end{array}\right] \\
\boldsymbol{F}_{\mathbf{2 4}}=\left[\begin{array}{cccc}
\cos \theta \cos \psi & 0 & 0 & 0 \\
\cos \theta \sin \psi & 0 & 0 & 0 \\
-\sin \psi & 0 & 0 & 0
\end{array}\right] \\
\boldsymbol{F}_{\mathbf{3 4}}=\left[\begin{array}{llll}
0 & 0 & 0 & 0 \\
0 & 0 & 0 & 0 \\
0 & 1 & 0 & 0
\end{array}\right]
\end{gathered}
$$

The shaping matrix $\boldsymbol{G}$ is written as follows :

$$
G=\left[\begin{array}{cccc}
G_{11} & 0_{3 \times 1} & 0_{3 \times 1} & 0_{3 \times 1} \\
0_{3 \times 1} & 0_{3 \times 1} & 0_{3 \times 1} & 0_{3 \times 1} \\
0_{3 \times 1} & G_{32} & G_{33} & G_{34} \\
0_{4 \times 1} & 0_{4 \times 1} & 0_{4 \times 1} & 0_{4 \times 1}
\end{array}\right]
$$

where $\boldsymbol{G}_{\mathbf{1 1}}=\left[\begin{array}{lll}\frac{\cos \theta \cos \psi}{M+h} & \frac{\cos \theta \sin \psi}{(N+h) \cos \varphi} & -\sin \theta\end{array}\right]^{T}, \boldsymbol{G}_{\mathbf{3 2}}=$ $\left[\begin{array}{lll}1 & 0 & 0\end{array}\right]^{T}, \boldsymbol{G}_{33}=\left[\begin{array}{lll}0 & 0 & 1\end{array}\right]^{T}, \boldsymbol{G}_{34}=\left[\begin{array}{lll}0 & 0 & 1\end{array}\right]^{T}$

The white noise vector $(\boldsymbol{u})$ is written as follows :

$$
\boldsymbol{\mu}=\left[\begin{array}{llll}
w_{v_{w S S}} & w_{\phi_{G S}} & w_{\theta_{G S}} & w_{\dot{\psi}_{Y R S}}
\end{array}\right]^{T}
$$

where $w_{v_{w S S}}$ is the white noise of WSS derived vehicle speed, $w_{\phi_{G S}}$ is the white noise of GS derived roll, $w_{\theta_{G S}}$ is the white noise of GS derived pitch, $w_{\dot{\psi}_{Y R S}}$ is the white noise of YRS.

The measurement model in EKF is generally expressed as follows:

$$
\boldsymbol{z}=\boldsymbol{H} \boldsymbol{\delta} \boldsymbol{x}+\boldsymbol{w}_{\boldsymbol{m}}
$$

where $\boldsymbol{z}$ is the measurement residual vector, $\boldsymbol{H}$ is the design matrix, and $\boldsymbol{w}_{\boldsymbol{m}}$ is the measurement noise vector.

The measurement residual vector for GNSS $\left(\boldsymbol{z}_{G N S S}\right)$ is the difference the position and velocity calculated by DR based on on-board vehicle sensors and the data acquired from GNSS receiver, as expressed in

$$
\mathbf{z}_{G N S S}=\left[\begin{array}{c}
\varphi \\
\lambda \\
h \\
v_{n} \\
v_{e} \\
v_{d}
\end{array}\right]_{D R}-\left[\begin{array}{c}
\varphi \\
\lambda \\
h \\
v_{n} \\
v_{e} \\
v_{d}
\end{array}\right]_{G N S S}
$$

Where the subscripts DR and GNSS denote the value calculated by DR based on on-board vehicle sensors and the data acquired from the GNSS receiver.

The design matrix for GNSS $\left(\boldsymbol{H}_{\boldsymbol{G N S S}}\right)$ is expressed in

$$
H_{G N S S}=\left[\begin{array}{llll}
I_{3 \times 3} & 0_{3 \times 3} & 0_{3 \times 3} & 0_{3 \times 4} \\
0_{3 \times 3} & I_{3 \times 3} & 0_{3 \times 3} & 0_{3 \times 4}
\end{array}\right]
$$

where $\boldsymbol{I}_{\boldsymbol{n} \times \boldsymbol{n}}$ is $\mathrm{n} \times \mathrm{n}$ identity matrix.

To prevent the drift error of yaw, the zero integrated heading rate (ZIHR) measurement update [14] is applied when the vehicle stationary. The measurement residual vector for ZIHR $\left(\mathbf{z}_{\boldsymbol{Z} I H R}\right)$ is given by

$$
\mathbf{z}_{\text {ZIHR }}=\psi_{k}-\psi_{k-1}
$$

where $\psi_{k}$ is the yaw angle at the current epoch, $\psi_{k-1}$ is the yaw angle at the previous epoch.

The design matrix vector for ZIHR is expressed in

$$
H_{Z I H R}=\left[\begin{array}{lllll}
\mathbf{0}_{1 \times 3} & 0_{1 \times 3} & 0_{1 \times 2} & 1 & 0_{1 \times 4}
\end{array}\right]
$$

\section{Performance evaluation of GNSS/on- board vehicle sensors integration algorithm}

To evaluate the performance of the proposed algorithm, a field test was conducted in a suburban of Daegu, Korea. Since the test vehicle (Figure 2) was equipped with ESC, the on-board vehicle sensors data were obtained from an in-vehicle control area network (CAN). The specifications of the on-board vehicles mounted on the test vehicle are described in Table 1. GNSS based position and velocity data were logged by the Novatel DL-V3GENERIC receiver. The GNSS positioning method was set to the point positioning used by automotive navigation system. According to the specification of the GNSS receiver, the accuracy of position and velocity are $1.5 \mathrm{~m}$ and $0.03 \mathrm{~m} / \mathrm{s}$, respectively. In order to assess the accuracy of navigation solution calculated by the proposed algorithm, the reference data were collected by a postprocessing of the data obtained from an Applanix's POS LV 520 system.

Table 1. Specification of on-board vehicle sensors

\begin{tabular}{|c|c|c|c|}
\hline Sensor & Output range & Resolution & Unit \\
\hline WSS & $0 \sim 511.75$ & 0.125 & $\mathrm{~km} / \mathrm{h}$ \\
\hline YRS & $-40.95 \sim 40.95$ & 0.01 & $\mathrm{deg} / \mathrm{s}$ \\
\hline GS & $-10.23 \sim 10.23$ & 0.01 & $\mathrm{~m} / \mathrm{s}^{2}$ \\
\hline
\end{tabular}




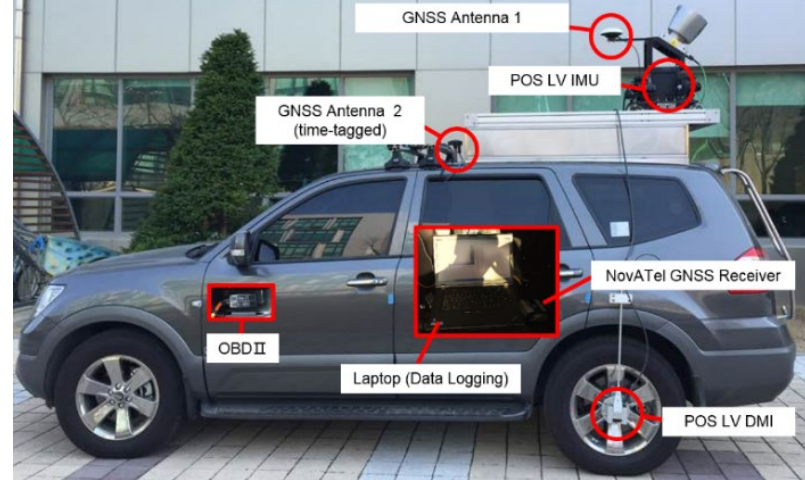

Fig. 2. Test Vehicle

The test trajectory for reference data is shown in Figure 3. The test trajectory covered various driving circumstance encountered during typical driving on urban roads with straight portions, turns, slopes, high speed, slow speeds, and jumping. The travelled distance of the trajectory is about $9.6 \mathrm{~km}$ for 38 minutes. Figure 4 shows the velocity profile in the body frame with the maximum speed being about $15 \mathrm{~m} / \mathrm{s}$. The number of tracked GNSS is more than five, as shown in Figure 5.

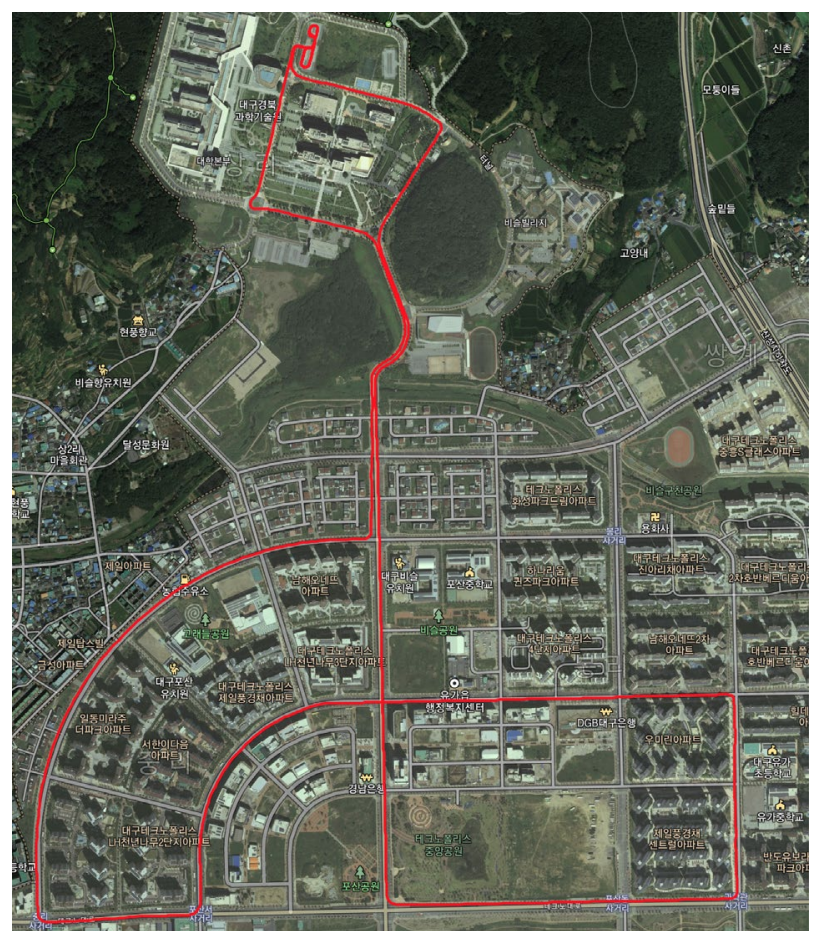

Fig. 3. Field test trajectory
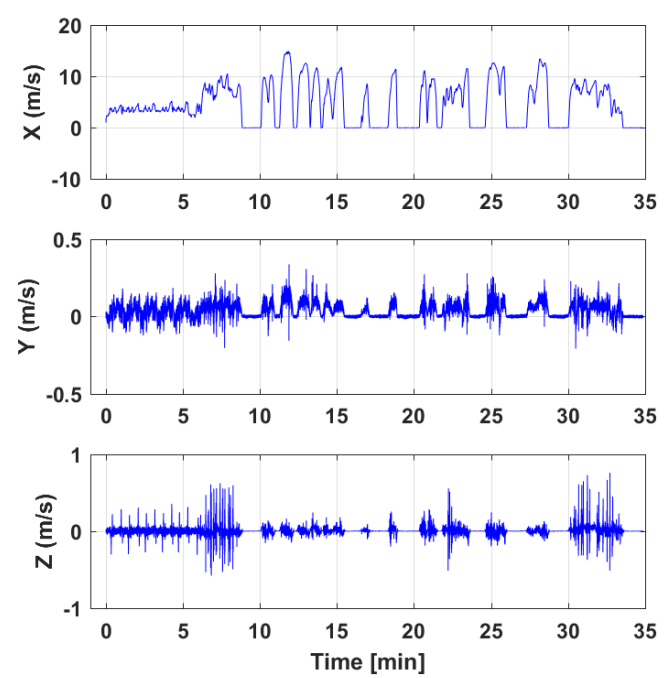

Fig. 4. Velocity in the body frane

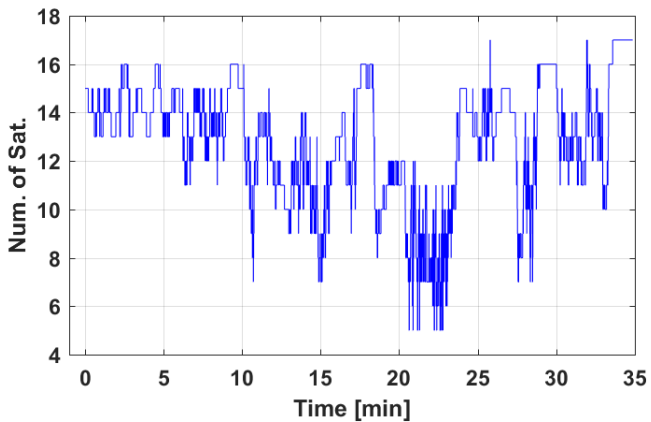

Fig. 5. Number of tracked GNSS

Table 2 summarize the statistics of the position error with respect to the reference data. It can be from Table 2 that the position error of the proposed algorithm is significantly decreased in comparison with the GNSS only solution. The standard deviation of position error along north and east direction is reduced by $70 \%$ and $43 \%$, respectivly, from $2.36 \mathrm{~m}$ and $1.91 \mathrm{~m}$ for the GNSS only solution to $0.70 \mathrm{~m}$ and $1.09 \mathrm{~m}$ for the proposed algorithm. Also, a decrease of standard deviation of position error along down direction for the proposed algorithm is $61 \%$ as compared to the GNSS only solution. The magnitude of position error of proposed algorithm is compared with the GNSS-only solution as shown in Figure 6. As seen in Figure 5 and 6 , the position error for GNSS-only solution is considerably affected by the number of tracked GNSS while aiding from the on-board sensors can provide a stable position during poor GNSS availability. 

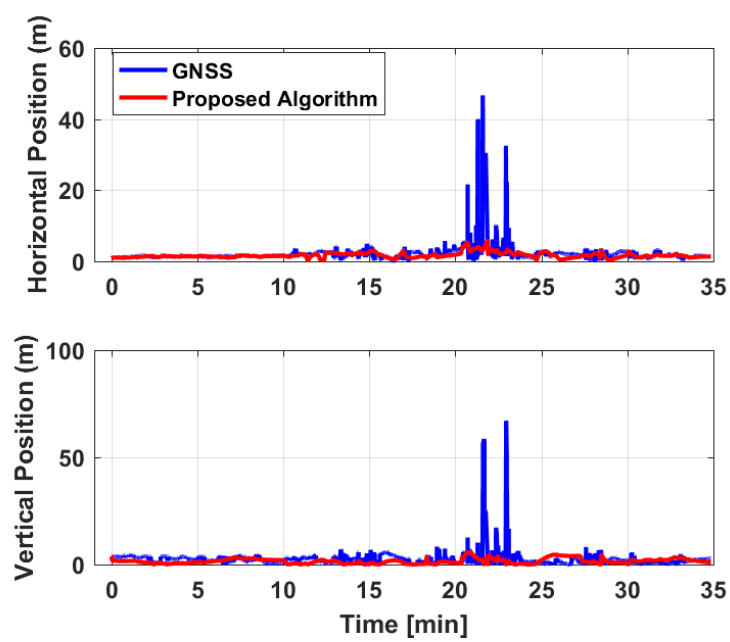

Fig. 6. The magnitude of position error for the proposed algorithm and the GNSS only solution

Table 2. The statistics of the postion error

\begin{tabular}{|c|c|c|c|c|}
\hline \multirow{2}{*}{$\begin{array}{c}\text { North } \\
(\mathrm{m})\end{array}$} & $\begin{array}{c}\text { Type } \\
\text { algorithm }\end{array}$ & $\begin{array}{c}-1.20 \sim \\
5.62\end{array}$ & 0.61 & 0.70 \\
\cline { 2 - 5 } & $\begin{array}{c}\text { GNSS } \\
\text { Only }\end{array}$ & $\begin{array}{c}-11.58 \sim \\
45.34\end{array}$ & 0.61 & 2.36 \\
\hline \multirow{2}{*}{$\begin{array}{c}\text { East } \\
(\mathrm{m})\end{array}$} & $\begin{array}{c}\text { Proposed } \\
\text { algorithm }\end{array}$ & $\begin{array}{c}-2.78 \sim \\
5.20\end{array}$ & 1.13 & 1.09 \\
\cline { 2 - 5 } & $\begin{array}{c}\text { GNSS } \\
\text { Only }\end{array}$ & $\begin{array}{c}-11.04 \sim \\
30.39\end{array}$ & 1.38 & 1.91 \\
\hline \multirow{2}{*}{$\begin{array}{c}\text { Down } \\
(\mathrm{m})\end{array}$} & $\begin{array}{c}\text { Proposed } \\
\text { algorithm }\end{array}$ & $\begin{array}{c}-4.36 \sim \\
5.79\end{array}$ & 0.95 & 1.74 \\
\cline { 2 - 5 } & GNSS & $-67.10 \sim$ & -1.93 & 4.45 \\
\hline
\end{tabular}

The statistics of the velocity error with respect to the reference data are given in Table 3 . The magnitude of velocity error of proposed algorithm is compared with the GNSS-only solution is shown in Figure 7. Compared to the GNSS only solution, the velocity error of the proposed algorithm is decreased by using on-board vehicle sensors. Especially, the performance of horizontal velocity can be further improved by the proposed algorithm with respect to the GNSS only solution.

Table 3. The statistics of the velocity error

\begin{tabular}{|c|c|c|c|c|}
\hline \multirow{2}{*}{} & Type & Range & Mean & $\begin{array}{c}\text { Standard } \\
\text { Deviation }\end{array}$ \\
\hline \multirow{2}{*}{$\begin{array}{c}\text { North } \\
(\mathrm{m} / \mathrm{s})\end{array}$} & $\begin{array}{c}\text { Proposed } \\
\text { algorithm }\end{array}$ & $\begin{array}{c}-0.55 \sim \\
0.94\end{array}$ & 0 & 0.09 \\
\cline { 2 - 5 } & $\begin{array}{c}\text { GNSS } \\
\text { Only }\end{array}$ & $\begin{array}{c}-1.45 \sim \\
2.20\end{array}$ & 0 & 0.10 \\
\hline \multirow{2}{*}{$\begin{array}{c}\text { East } \\
(\mathrm{m} / \mathrm{s})\end{array}$} & $\begin{array}{c}\text { Proposed } \\
\text { algorithm }\end{array}$ & $\begin{array}{c}-3.56 \sim \\
0.90\end{array}$ & 0 & 0.12 \\
\cline { 2 - 5 } & \begin{tabular}{c} 
OnSS \\
\multirow{2}{*}{$\begin{array}{c}\text { Down } \\
(\mathrm{m} / \mathrm{s})\end{array}$}
\end{tabular} & $\begin{array}{c}-3.77 \sim \\
1.43\end{array}$ & 0 & 0.12 \\
\cline { 2 - 5 } & algorithm & $\begin{array}{c}-1.45 \sim \\
1.34\end{array}$ & 0.04 & 0.12 \\
\hline
\end{tabular}
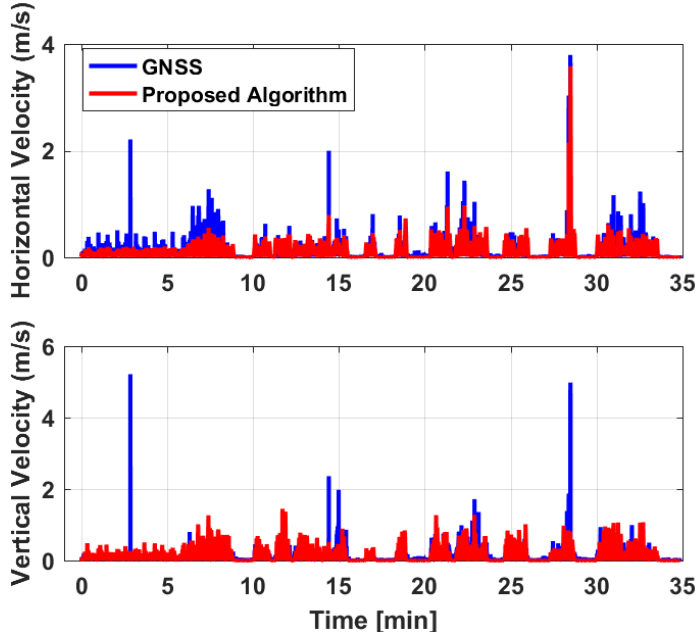

Fig. 7. The magnitude of position velocity for the proposed algorithm and the GNSS only solution

\section{Conclusion}

In this paper, GNSS and on-board vehicle sensors (two wheel speed sensors, a yaw rate sensor, and two gravity sensors) integrated vehicle positioning algorithm was developed to overcome the limitation of GNSS based technique. On-board sensors were applied for three dimensional dead reckoning to compute the navigation solutions without GNSS. And the GNSS/on-board vehicle sensors integration was implemented by EKF through a loosely coupled mode.

Performance was evaluated through tests carried out in real driving trajectory including various GNSS signals reception environments. The result indicates that the GNSS combined on-board vehicle sensors integration can enhance the positioning accuracy as compared with using only GNSS. Also, we found that GNSS/on-board vehicle sensors integration can provide a stable position during poor GNSS availability. Therefore, the proposed algorithm can be an alternative solution to compensate the limitation of the GNSS positioning technique, without the use of a low-cost inertial measurement unit.

\section{Acknowledgements}

This work was supported by the DGIST R\&D Program of the Ministry of Science, ICT \& Technology of Korea (18-NT-01).

\section{References}

1. K. Jo, K. Chu, and M. Sunwoo, IEEE trans. Intell. Transp. Sys., 13, 1 (2012)

2. I. Skog, and P. Handel, IEEE trans. Intell. Transp. Sys., 13, 1 (2012)

3. X. Li, R. Jiang, X. Song, and B. Li, Journal of Sensors, 2017, 11 (2017)

4. D. Barbosa, A. Lopes, and R. E. Araujo, IECON'16, pp.1049-1054 (2016). 
5. J. Kubo, T. Kindo, A. Ito, and S. Sugimoto, ION GPS 1999, pp.555-564 (1999).

6. P. Bonnifait, P. Bouron, D. Meizel, and P. Crubille, Journal of Navigation, 53, 6 (2003).

7. J. Georgy, A. Noureldim, M.J. Korenberg, and M. M. Bayoumi, IEEE Transaction on Vehicular Technology, 59, 2 (2010).

8. J. Gao, Development of a precise GPS/INS/on-board vehicle sensors integrated vehicular positioning system (2007).

9. P. Bonnifait, P. Bouron, P. Crubille, and D. Meizel, 2001 ICRA, 2, pp.1597-1602 (2001).

10. C.R. Carlson, Estimation with applications for automobile dead reckoning and control (2004).

11. U. Iqbal, Multi-sensor data fusion for vehicular navigation applications (2012).

12. S. Razaei, and R. Sengupta, IEEE Transaction on Vehicular Technology, 15, 6 (2007).

13. K. Jo, K. Chu, and M. Sunwoo, IEEE Transactions on Intelligent Transportation System, 13, 1 (2012).

14. E. Shin, Estimation techniques for low-cost inertial navigation (2005). 